\title{
A Mellin Transform Approach to the Pricing of Options with Default Risk
}

\author{
Sun-Yong Choi ${ }^{1} \cdot$ Sotheara Veng ${ }^{2} \cdot$ Jeong-Hoon $\mathrm{Kim}^{3} \cdot \mathrm{Ji}-\mathrm{Hun}$ Yoon $^{4}$
}

Accepted: 10 April 2021 / Published online: 26 April 2021

(C) The Author(s), under exclusive licence to Springer Science+Business Media, LLC, part of Springer Nature 2021

\begin{abstract}
The stochastic elasticity of variance model introduced by Kim et al. (Appl Stoch Models Bus Ind 30(6):753-765, 2014) is a useful model for forecasting extraordinary volatility behavior which would take place in a financial crisis and high volatility of a market could be linked to default risk of option contracts. So, it is natural to study the pricing of options with default risk under the stochastic elasticity of variance. Based on a framework with two separate scales that could minimize the number of necessary parameters for calibration but reflect the essential characteristics of the underlying asset and the firm value of the option writer, we obtain a closed form approximation formula for the option price via double Mellin transform with singular perturbation. Our formula is explicitly expressed as the Black-Scholes formula plus correction terms. The correction terms are given by the simple derivatives of the Black-Scholes solution so that the model calibration can be done very fast and effectively.
\end{abstract}

Keywords Mellin transform - Stochastic elasticity of variance $\cdot$ Option - Default risk

Ji-Hun Yoon

yssci99@pusan.ac.kr

1 Department of Financial Mathematics, Gachon University, Gyeonggi 13120, Republic of Korea

2 Graduate School of Science, Royal University of Phnom Penh, Phnom Penh 12156, Kingdom of Cambodia

3 Department of Mathematics, Yonsei University, Seoul 03722, Republic of Korea

4 Department of Mathematics, Pusan National University, Busan 46241, Republic of Korea 


\section{Introduction}

In general, derivatives traded on stock exchanges are considered to be no default risk. However, several derivatives such as currency options, options on precious metals, interest rate swaps, or credit default swaps, still have been traded in overthe-counter(OTC) market where there is no the exchange or clearing corporation. Therefore, many buyers of the related derivatives are vulnerable to the default risk. In other words, the option holder is always apt to have counterparty credit risks since the option writer of the counterparty may not fulfil the appointed contracts at the maturity. So, we need to take account of the default risk of the contingent claim for pricing the options on the defaultable instruments in such OTC market. the option is called a 'vulnerable option' when the option buyer is exposed to the option writer's default risk which is an example of a counterparty risk. Financial crises such as the Global Financial Crisis, Eurozone crisis and COVID-19 pandemic have brought the issue of counterparty risk to academic researchers and practitioners. We also note that counterparty risks are an important risk factor recognized by Basel III. Especially, the recent economic crisis caused by the COVID-19 pandemic has increased the importance of the default risk on the derivatives. According to BIS report, ${ }^{1}$ the gross market value of OTC derivatives rose from $\$ 11.6$ trillion to $\$ 15.5$ trillion during the first half of 2020. Correspondingly, gross credit exposures reached $\$ 3.2$ trillion at end-June 2020, which is the largest increase since 2009. The largest growth indicates that many holders of the option contracts should be seriously aware of the default possibilities.

The pricing of European vulnerable options has been already proposed by Johnson and Stulz (1987). Since then, several works have been reported by researchers on the pricing of vulnerable options. For example, Klein (1996) derived a formula for European vulnerable option when a proportion of nominal claim is paid out in default depending on the amount of other liabilities as well as the assets of the counterparty. In that study, the value of the asset of the counterparty is assumed to follow a geometric Brownian motion. Hung and Liu (2005) obtained a vulnerable option pricing formula under an incomplete market condition. The advantage of the formula is that it can evaluate nontraded assets underlying vulnerable options. Yang et al. (2014) assumed that volatility of the asset value follows a fast mean-reverting Ornstein-Ulenbeck (OU) process and derived an analytic formula for European vulnerable option. Yoon and Kim (2015) derived an explicit closed form formula of European vulnerable option with stochastic interest rates following the Hull-White interest rate process.

In this study, we evaluate the price of vulnerable options based on the stochastic of elasticity of variance (SEV) model introduced by Kim et al. (2014). The SEV model forecasts market's volatility more accurately than the constant elasticity of variance $(\mathrm{CEV})$ model so that investors can employ a dynamic investment strategy reducing the risk more effectively. In particular, it is a useful model for extraordinary volatility behavior which would take place in a financial crisis. Refer to Kim et al. (2015). The model consists of two separate scales in such a way that

\footnotetext{
${ }^{1}$ OTC derivative statistics at end-June 2020.
} 
there are the following important merits. The first one is that the number of necessary parameters for calibration is minimized while the model well reflects the essential characteristics of the underlying asset and the firm value of the option writer. The second one is that singular perturbation technique can be implemented to extract essential information for the properties of the option price. The SEV model has been used to the pricing of other types of derivatives. For example, Yoon and Park (2016) and Yoon et al. (2013) evaluated turbo warrant options and perpetual American options, respectively. It could be hybridized with a pure stochastic volatility model such as the Heston model (1993) as done by Choi et al. (2016).

A Mellin transform is an integral transform that can be thought of as the multiplicative version of the two-sided Laplace transform. The Mellin transform can be a very useful tool for the transformation of partial differential equations (PDEs) as described in Brychkov (1992). In general, the Mellin transform technique, if it is plausible for the pricing of a given option, would not require the complexity of the calculation as appeared in the probabilistic approaches. The Mellin transform technique has been used for pricing financial derivatives in Panini and Srivastav (2004), Panini and Srivastav (2005), Brychkov (1992), Frontczak and Schöbel (2010), Frontczak (2013), Jeon et al. (2016), Jeon et al. (2017) and Guardasoni et al. (2020). In this study, we first employ singular perturbation technique to obtain PDEs for the vulnerable option price and then exploit the double Mellin transform introduced by Krapivsky and Ben-Naim (1994) to derive an analytic solution for each PDE. The double Mellin transform provides an explicit closed form formula.

Model calibration in financial engineering is as important as the model itself. Calibration is equivalent to identifying the parameter values so that the model can reproduce market prices as accurately as possible. The speed as well as the accuracy of calibration is crucial because practitioners use the calibrated parameters to price complicated derivatives and to work out trading strategies. A main contribution of this paper is that our option price formula is given in a relatively simple closed form explicitly. It is the Black-Scholes price plus correction terms that can be calculated by simply taking derivatives of the Black-Scholes formula. In this sense, our pricing formula is better than the well-known formula under the Heston model introduced by Heston (1993), one of the most popular stochastic volatility models for pricing derivatives, which is given analytically but requires a numerical method to compute the complex integral. Actually, the semi-analytical pricing formula for the European vanilla options by Heston model obtained by the use of Fouier transform techniques comes up with the instability and a great deal of computing time in obtaining the solutions, including the problem of the model calibrations. So, the existence of the closed solution for the option pricing is very important because it directly affects the computing time in implementing the option's data fitting (or the calibration of option's model) and the numerical stability related to the pricing accuracy. This paper shows that the SEV model with the Mellin transform leads to a better analytical solution for pricing derivatives than the Heston model with the Fourier transform in terms of the stability (or the accuracy) and the computing time.

The structure of the paper is organized as follows. Section 2 gives a brief review of the SEV model. In Sect. 3, we apply multiscale asymptotic analysis to 
constructing an explicit approximation of the European vulnerable option price. The explicit analytic solution is given by the Melling transform technique in Sect. 4. Section 5 is committed to investigating the sensitivity of the vulnerable option value with regard to the model parameters and verifying the accuracy and efficiency of the approximate formula. Section 6 provides concluding remarks.

\section{Model Formulation}

We first set out to write the SEV model given by Kim et al. (2014). In a market measure, the underlying asset price satisfies

$$
\begin{gathered}
d X_{t}=\mu_{x} X_{t} d t+\sigma_{x} X_{t}^{\theta_{t}} d W_{t}^{x}, \quad \theta_{t}=1-\sqrt{\delta} f\left(Y_{t}\right), \\
d Y_{t}=\frac{1}{\epsilon}\left(m-Y_{t}\right) d t+\frac{1}{\sqrt{\epsilon}} v \sqrt{2} d W_{t}^{y}
\end{gathered}
$$

where $\mu_{x}, \sigma_{x}$ and $m$ are constants and $\epsilon$ and $\delta$ are small positive parameters and $d\left\langle W^{x}, W^{y}\right\rangle_{t}=\rho_{x y} d t$. The elasticity parameter $\theta_{t}$ is fluctuated by financial circumstance and the magnitude of its fluctuation is shown to be small as in Kim et al. (2015).

Next, let $V_{t}$ be the market value (firm value) of the assets of the option writer. Its dynamics is given by

$$
d V_{t}=\mu_{v} V_{t} d t+\sigma_{v} V_{t} d W_{t}^{v},
$$

where $\mu_{v}$ and $\sigma_{v}$ are constants and $d\left\langle W^{x}, W^{v}\right\rangle_{t}=\rho_{x v} d t$ and $d\left\langle W^{v}, W^{y}\right\rangle_{t}=\rho_{v y} d t$. We note here the comment from Hoffmann (2018) that the value of firms should not be based on just shareholders but should include all groups of stakeholders.

By using the changes of Brownian motions

$$
\begin{aligned}
& d W_{t}^{x *}=d W_{t}^{x}+\frac{\mu_{x}-r}{\sigma_{x} X_{t}^{-\sqrt{\delta} f\left(Y_{t}\right)}} d t, \\
& d W_{t}^{v *}=d W_{t}^{v}+\frac{\mu_{v}-r}{\sigma_{v}} d t,
\end{aligned}
$$

we can transform the market measure to an equivalent martingale measure $Q^{*}$ under which the dynamics of the processes $X_{t}, Y_{t}$ and $V_{t}$ becomes

$$
\begin{gathered}
d X_{t}=r X_{t} d t+\sigma_{x} X_{t}^{1-\sqrt{\delta} f\left(Y_{t}\right)} d W_{t}^{x *} \\
d Y_{t}=\left[\frac{1}{\epsilon}\left(m-Y_{t}\right)-\frac{1}{\sqrt{\epsilon}} v \sqrt{2} \Lambda\left(X_{t}, Y_{t}\right)\right] d t+\frac{1}{\sqrt{\epsilon}} v \sqrt{2} d W_{t}^{y *}, \\
d V_{t}=r V_{t} d t+\sigma_{v} V_{t} d W_{t}^{v *}
\end{gathered}
$$

where $r$ is a risk-free interest rate and $\Lambda$ is the market price of risk. The correlation structure of the Brownian motions $W_{t}^{x *}, W_{t}^{y *}$ and $W^{\nu *}$ is given by 


$$
\begin{aligned}
& d\left\langle W^{x *}, W^{y *}\right\rangle_{t}=\rho_{x y} d t, \\
& d\left\langle W^{x *}, W^{v *}\right\rangle_{t}=\rho_{x v} d t, \\
& d\left\langle W^{v *}, W^{y *}\right\rangle_{t}=\rho_{v y} d t .
\end{aligned}
$$

We need to give a restriction on $f$ in order to guarantee the regularity condition. It is assumed that $f$ is bounded and $\delta$ is small $(0<\delta \ll 1)$ and positive enough to satisfy the inequality $0 \leq f<\frac{1}{\sqrt{\delta}}$ apart from the absorbing boundary condition at $t=0$. Refer to Delbaen and Shirakawa (2002).

In this study, we consider a European vulnerable call option with its payoff given by

$$
h\left(X_{t}, V_{t}\right)=\left(X_{T}-K\right)^{+}\left(1_{\left\{V_{T} \geq D^{*}\right\}}+1_{\left\{V_{T}<D^{*}\right\}} \frac{(1-\alpha) V_{T}}{D}\right) .
$$

Here, $K$ is the strike price of the option. $D, D^{*}$, and $\alpha$ are related with the credit loss event in the vulnerable option. $D^{*}$ is the critical value such that default occurs if the value of the option writer's assets, $V_{T}$, is less than $D^{*}$. In practice, this term is stochastic rather than constant but it is assumed to be a constant in this paper. $D$ is the value of total liabilities. $D^{*}$ can be less than $D$ because of an additional liability owing to the possibility of a counterparty keeping operation. $\alpha$ represents the deadweight costs related with bankruptcy expressed as a percentage of the value of the assets of the option writer. If $V_{T}$ is larger than or equal to the default boundary $D^{*}$, no credit loss occurs and the payoff becomes full payment $h\left(X_{t}\right)=\left(X_{T}-K\right)^{+}$. If not, the proportion $\frac{(1-\alpha) V_{T}}{D}$ of the nominal claim $\left(X_{T}-K\right)^{+}$is paid out.

Under the risk neutral measure $Q^{*}$, the price of a European vulnerable option is determined by the formula

$$
P^{\delta, \epsilon}(t, x, y, v)=E^{*}\left[e^{-r(T-t)} h\left(X_{T}, V_{T}\right) \mid X_{t}=x, Y_{t}=y, V_{t}=v\right],
$$

where $r$ is the risk free rate of interest over $[t, T]$.

Using the Feyman-Kac formula (cf. Oksendal 2013),$P^{\delta, \epsilon}(t, x, y, v)$ solves the partial differential equation (PDE)

$$
\begin{aligned}
& \frac{\partial P^{\delta, \epsilon}}{\partial t}+r x \frac{\partial P^{\delta, \epsilon}}{\partial x}+\frac{1}{2} \sigma_{x}^{2} x^{2(1-f(y) \sqrt{\delta})} \frac{\partial^{2} P^{\delta, \epsilon}}{\partial x^{2}}+r v \frac{\partial P^{\delta, \epsilon}}{\partial v}+\frac{1}{2} \sigma_{v}^{2} v^{2} \frac{\partial^{2} P^{\delta, \epsilon}}{\partial v^{2}} \\
& \quad+\rho_{x v} \sigma_{x} \sigma_{v} x^{1-f(y) \sqrt{\delta}} v \frac{\partial^{2} P^{\delta, \epsilon}}{\partial x \partial v}-r P^{\delta, \epsilon} \\
& +\frac{1}{\sqrt{\epsilon}}\left(\rho_{x y} \sigma_{x} v \sqrt{2} x^{1-f(y) \sqrt{\delta}} \frac{\partial^{2} P^{\delta, \epsilon}}{\partial x \partial y}+\rho_{v y} \sigma_{v} v \sqrt{2} v \frac{\partial^{2} P^{\delta, \epsilon}}{\partial v \partial y}-v \sqrt{2} \Lambda(x, y) \frac{\partial P^{\delta, \epsilon}}{\partial y}\right) \\
& +\frac{1}{\epsilon}\left(v^{2} \frac{\partial^{2} P^{\delta, \epsilon}}{\partial y^{2}}+(m-y) \frac{\partial P^{\delta, \epsilon}}{\partial y}\right)=0, \quad t<T,
\end{aligned}
$$

where $P^{\delta, \epsilon}(T, x, y, v)=h(x, v)=(x-K)^{+}\left(1_{\left\{v \geq D^{*}\right\}}+1_{\left\{v<D^{*}\right\}} \frac{(1-\alpha) v}{D}\right)$.

We note that the PDE problem (2.8) has no analytic solution for general $f$. However, for small parameters $\epsilon$ and $\delta$, one can proceed with asymptotic analysis to 
obtain an explicit approximate solution, which will be carried out in the next section.

\section{Multiscale Analysis}

Now, we apply multiscale asymptotic analysis developed by Fouque et al. (2011) to derive an approximate formula for $P^{\delta, \epsilon}$ satisfying (2.8). We denote by $\langle\cdot\rangle$ the averaging with respect to the invariant distribution of the process $Y_{t}$.

First, we define the differential operator $\mathcal{L}^{\delta, \epsilon}$ as

$$
\begin{gathered}
\mathcal{L}^{\delta, \epsilon}=\frac{1}{\epsilon} \mathcal{L}_{0}+\frac{1}{\sqrt{\epsilon}} \mathcal{L}_{1}^{\delta}+\mathcal{L}_{2}^{\delta} \\
\mathcal{L}_{0}:=v^{2} \frac{\partial^{2}}{\partial y^{2}}+(m-y) \frac{\partial}{\partial y} \\
\mathcal{L}_{1}^{\delta}:=\rho_{x y} \sigma_{x} v \sqrt{2} x^{1-f(y) \sqrt{\delta}} \frac{\partial^{2}}{\partial x \partial y}+\rho_{v y} \sigma_{v} v \sqrt{2} v \frac{\partial^{2}}{\partial v \partial y}-v \sqrt{2} \Lambda(x, y) \frac{\partial}{\partial y}, \\
\left.\mathcal{L}_{2}^{\delta}:=\frac{\partial}{\partial t}+r x \frac{\partial}{\partial x}+\frac{1}{2} \sigma_{x}^{2} x^{2(1-f(y) \sqrt{\delta})} \frac{\partial^{2}}{\partial x^{2}}+r v \frac{\partial}{\partial v}+\frac{1}{2} \sigma_{v}^{2} v^{2} \frac{\partial^{2}}{\partial v^{2}}+\rho_{x v} \sigma_{x} \sigma_{v} x^{1-f(y) \sqrt{\delta}} v \frac{\partial^{2}}{\partial x \partial v}-r .3\right)
\end{gathered}
$$

As the operators $\mathcal{L}_{1}^{\delta}$ and $\mathcal{L}_{2}^{\delta}$ depend on $\delta$, we use Taylor expansion with respect to $\sqrt{\delta}$ to express the operators $\mathcal{L}_{1}^{\delta}$ and $\mathcal{L}_{2}^{\delta}$ in powers of $\sqrt{\delta}$ as follows:

$$
\begin{gathered}
\mathcal{L}_{1}^{\delta}=\mathcal{L}_{10}+\sqrt{\delta} \mathcal{L}_{11}+\delta \mathcal{L}_{12}+\cdots \\
\mathcal{L}_{2}^{\delta}=\mathcal{L}_{20}+\sqrt{\delta} \mathcal{L}_{21}+\delta \mathcal{L}_{22}+\cdots \\
\mathcal{L}_{10}:=\rho_{x y} \sigma_{x} v \sqrt{2} x \frac{\partial^{2}}{\partial x \partial y}+\rho_{v y} \sigma_{v} v \sqrt{2} v \frac{\partial^{2}}{\partial v \partial y}-v \sqrt{2} \Lambda(x, y) \frac{\partial}{\partial y} \\
\mathcal{L}_{11}:=-\rho_{x y} \sigma_{x} v \sqrt{2} f(y) x \ln x \frac{\partial^{2}}{\partial x \partial y} \\
\mathcal{L}_{12}:=\rho_{x y} \sigma_{x} v \sqrt{2} \frac{(f(y) \ln x)^{2}}{2} x \frac{\partial^{2}}{\partial x \partial y} \\
\mathcal{L}_{20}:=\frac{\partial}{\partial t}+r\left(x \frac{\partial}{\partial x}+v \frac{\partial}{\partial v}-\cdot\right)+\frac{1}{2} \sigma_{x}^{2} x^{2} \frac{\partial^{2}}{\partial x^{2}}+\frac{1}{2} \sigma_{v}^{2} v^{2} \frac{\partial^{2}}{\partial v^{2}}+\rho_{x v} \sigma_{x} \sigma_{v} x v \frac{\partial^{2}}{\partial x \partial v}
\end{gathered}
$$




$$
\begin{gathered}
\mathcal{L}_{21}:=-\sigma_{x}^{2} f(y) x^{2} \ln x \frac{\partial^{2}}{\partial x^{2}}-\rho_{x v} \sigma_{x} \sigma_{v} f(y) x v \ln x \frac{\partial^{2}}{\partial x \partial v}, \\
\mathcal{L}_{22}:=\sigma_{x}^{2}(f(y) \ln x)^{2} x^{2} \frac{\partial^{2}}{\partial x^{2}}+\rho_{x v} \sigma_{x} \sigma_{v} \frac{(f(y) \ln x)^{2}}{2} x v \frac{\partial^{2}}{\partial x \partial v} .
\end{gathered}
$$

Notice that $\mathcal{L}_{20}$ is exactly the operator corresponding to the Black-Scholes model with constant volatility for vulnerable European options. We denote it by $\mathcal{L}_{B S}$ from now on.

We are interested in the solution of the PDE (2.8) of the form $P^{\delta, \epsilon}=$ $P_{0}^{\epsilon}+\sqrt{\delta} P_{1}^{\epsilon}+\delta P_{2}^{\epsilon}+\cdots$. Substituting this into (2.8) yields the following hierarchy of PDEs:

$$
\begin{gathered}
\delta^{0}:\left(\frac{1}{\epsilon} \mathcal{L}_{0}+\frac{1}{\sqrt{\epsilon}} \mathcal{L}_{10}+\mathcal{L}_{20}\right) P_{0}^{\epsilon}=0 \\
\delta^{\frac{1}{2}}:\left(\frac{1}{\epsilon} \mathcal{L}_{0}+\frac{1}{\sqrt{\epsilon}} \mathcal{L}_{10}+\mathcal{L}_{20}\right) P_{1}^{\epsilon}+\left(\frac{1}{\sqrt{\epsilon}} \mathcal{L}_{11}+\mathcal{L}_{21}\right) P_{0}^{\epsilon}=0 \\
\delta:\left(\frac{1}{\epsilon} \mathcal{L}_{0}+\frac{1}{\sqrt{\epsilon}} \mathcal{L}_{10}+\mathcal{L}_{20}\right) P_{2}^{\epsilon}+\left(\frac{1}{\sqrt{\epsilon}} \mathcal{L}_{11}+\mathcal{L}_{21}\right) P_{1}^{\epsilon}+\left(\frac{1}{\sqrt{\epsilon}} \mathcal{L}_{12}+\mathcal{L}_{22}\right) P_{0}^{\epsilon}=0 .
\end{gathered}
$$

Then we plug the expansions

$$
P_{i}^{\epsilon}=P_{i, 0}+\sqrt{\epsilon} P_{i, 1}+\epsilon P_{i, 2}+\epsilon \sqrt{\epsilon} P_{i, 3}+\cdots \quad(i=0,1,2)
$$

into (3.13) and obtain

$$
\begin{aligned}
P^{\delta, \epsilon}= & P_{0,0}+\sqrt{\delta} P_{1,0}+\sqrt{\epsilon} P_{0,1}+\delta P_{2,0}+\sqrt{\delta \epsilon} P_{1,1}+\epsilon P_{0,2}+\delta \sqrt{\delta} P_{3,0} \\
& +\sqrt{\delta} \epsilon P_{1,2}+\sqrt{\epsilon} \delta P_{2,1}+\epsilon \sqrt{\epsilon} P_{0,3}+\cdots
\end{aligned}
$$

assuming that $\epsilon$ and $\delta$ satisfy the order condition $\epsilon \ll \delta \ll \sqrt{\epsilon}$.

We obtain the following Propositions 3.1 and 3.2.

Proposition $\quad 3.1$ Let $P_{0,0}(T, x, y, v)=h(x, v) \quad$ and $\quad P_{0, i}(T, x, y, v)=0 \quad$ for $i=0,1,2 \ldots$. Assume that the partial derivative of $P_{0, i}$ with respect to $y$ does not grow as much as $\frac{\partial P_{0, i}}{\partial y} \sim e^{\frac{1}{y^{2}}}$ for $i=0,1,2 \ldots$ when $y$ goes to infinity. Then $P_{0, i}=0$ for $i=0,1,2 \ldots$ and it is independent of $y$ and $P_{0,0}$ satisfies the PDE

$$
\begin{aligned}
\mathcal{L}_{B S} P_{0,0}(t, x, v) & =0, \quad t<T, \\
P_{0,0}(T, x, v) & =h(x, v),
\end{aligned}
$$


whose solution is given by

$$
\begin{aligned}
P_{0,0}(t, x, v) & =x \mathcal{N}_{2}\left(a_{1}, a_{2}, \rho_{x v}\right)-K e^{-r(T-t)} \mathcal{N}_{2}\left(b_{1}, b_{2}, \rho_{x v}\right) \\
& +\frac{(1-\alpha) v}{D}\left(x e^{\left(r+\rho_{x v} \sigma_{x} \sigma_{v}\right)(T-t)} \mathcal{N}_{2}\left(c_{1}, c_{2},-\rho_{x v}\right)-K \mathcal{N}_{2}\left(d_{1}, d_{2},-\rho_{x v}\right)\right), \\
\mathcal{N}_{2}\left(n_{1}, n_{2}, \rho_{x v}\right) & =\frac{1}{2 \pi \sqrt{1-\rho_{x v}^{2}}} \int_{-\infty}^{n_{1}} \int_{-\infty}^{n_{2}} e^{-\frac{1}{2\left(1-\rho_{x v}^{2}\right.}\left(p^{2}-2 \rho_{x v} p q+q^{2}\right)} d p d q, \\
a_{1} & =\frac{1}{\sigma_{x} \sqrt{T-t}}\left[\ln \left(\frac{x}{K}\right)+\left(r+\frac{\sigma_{x}^{2}}{2}\right)(T-t)\right], \\
a_{2} & =\frac{1}{\sigma_{v} \sqrt{T-t}}\left[\ln \left(\frac{v}{D^{*}}\right)+\left(r-\frac{\sigma_{v}^{2}}{2}+\rho_{x v} \sigma_{x} \sigma_{v}\right)(T-t)\right], \\
b_{1} & =\frac{1}{\sigma_{x} \sqrt{T-t}}\left[\ln \left(\frac{x}{K}\right)+\left(r-\frac{\sigma_{x}^{2}}{2}\right)(T-t)\right], \\
b_{2} & =\frac{1}{\sigma_{v} \sqrt{T-t}}\left[\ln \left(\frac{v}{D^{*}}\right)+\left(r-\frac{\sigma_{v}^{2}}{2}\right)(T-t)\right], \\
c_{1} & =\frac{1}{\sigma_{x} \sqrt{T-t}}\left[\ln \left(\frac{x}{K}\right)+\left(r+\frac{\sigma_{x}^{2}}{2}+\rho_{x v} \sigma_{x} \sigma_{v}\right)(T-t)\right], \\
c_{2} & =-\frac{1}{\sigma_{v} \sqrt{T-t}}\left[\ln \left(\frac{v}{D^{*}}\right)+\left(r+\frac{\sigma_{v}^{2}}{2}+\rho_{x v} \sigma_{x} \sigma_{v}\right)(T-t)\right], \\
d_{1} & =\frac{1}{\sigma_{x} \sqrt{T-t}}\left[\ln \left(\frac{x}{K}\right)+\left(r-\frac{\sigma_{x}^{2}}{2}+\rho_{x v} \sigma_{x} \sigma_{v}\right)(T-t)\right], \\
d_{2} & =-\frac{1}{\sigma_{v} \sqrt{T-t}}\left[\ln \left(\frac{v}{D^{*}}\right)+\left(r+\frac{\sigma_{v}^{2}}{2}\right)(T-t)\right] .
\end{aligned}
$$

Proof Plugging the expansion $P_{0}^{\epsilon}$ into the PDE (3.13), we obtain the recursive relation

$$
\mathcal{L}_{0} P_{0, k}+\mathcal{L}_{10} P_{0, k-1}+\mathcal{L}_{B S} P_{0, k-2}=0, \quad k=0,1,2, \ldots,
$$

where $P_{0,-1}=P_{0,-2}=0$ is defined for consistency.

If $k=0$, we have the ordinary differential equation (ODE) $\mathcal{L}_{0} P_{0,0}=0$. By solving this ODE, one can find

$$
P_{0,0}(t, x, y, v)=K_{1}(t, x, v) \int_{-\infty}^{y} e^{(z-m)^{2} / 2 v^{2}} d z+K_{2}(t, x, v)
$$

for some functions $K_{1}$ and $K_{2}$ independent of $y$. From the assumed growth condition, $K_{1}$ must disappear. So, $P_{0,0}$ is independent of the $y$ variable, that is, $P_{0,0}=$ $P_{0,0}(t, x, v)$ and thus $\mathcal{L}_{10} P_{0,0}=0$. Then (3.18) for $k=1$ yields the ODE $\mathcal{L}_{0} P_{0,1}=0$, meaning that $P_{0,1}$ is independent of $y$, that is, $P_{0,1}=P_{0,1}(t, x, v)$ and thus $\mathcal{L}_{10} P_{0,1}=0$. Then (3.18) with $k=2$ leads to the PDE 


$$
\mathcal{L}_{0} P_{0,2}+\mathcal{L}_{B S} P_{0,0}=0 .
$$

Since (3.20) is a Poisson equation for $P_{0,2}$ in terms of $\mathcal{L}_{0}$, one can apply the solvability condition on the source term $\mathcal{L}_{B S} P_{0,0}$ from the Fredholm alternative. Therefore, we obtain (3.16) and the solution of it is given by (3.17) from Theorem 1 in Yoon and Kim (2015).

Now, from (3.16) and (3.20), the $\operatorname{ODE} \mathcal{L}_{0} P_{0,2}=0$ is satisfied so that $P_{0,2}=P_{0,2}(t, x, v)$, which yields the PDE $\mathcal{L}_{10} P_{0,2}=0$. Then (3.18) for $k=3$ leads to $\mathcal{L}_{0} P_{0,3}+\mathcal{L}_{B S} P_{0,1}=0$, which is a Poisson equation of $y$ in terms of $\mathcal{L}_{0}$. Again, the solvability condition leads to the $\operatorname{PDE} \mathcal{L}_{B S} P_{0,1}=0$. The solution of this equation with the final condition $P_{0,1}(T, x, v)=0$ is $P_{0,1}=0$. Similarly, for $k=4$, (3.18) becomes $\mathcal{L}_{0} P_{0,4}+\mathcal{L}_{B S} P_{0,2}=0$, which leads to $\mathcal{L}_{B S} P_{0,2}=0$. Then, from the final condition $P_{0,2}(T, x, v)=0$, the solution is exactly $P_{0,2}=0$. Then, by mathematical induction, one can have the result $P_{0, i}=0$ for $i=1,2, \ldots$

Proposition 3.2 Assume that the partial derivatives of $P_{1,0}$ and $P_{1,1}$ with respect to $y$ do not grow as much as $\frac{\partial P_{1, i}}{\partial y} \sim e^{\frac{1}{y^{2}}}$ for $i=0,1$ as $y$ goes to infinity. Then $P_{1,0}$ and $P_{1,1}$ are the independents of $y$ and $P_{1,0}^{\delta}=\sqrt{\delta} P_{1,0}$ satisfies the PDE

$$
\begin{aligned}
\mathcal{L}_{B S} P_{1,0}^{\delta} & =\mathcal{A}_{1,0} P_{0}, \quad t<T \\
P_{1,0}^{\delta}(T, x, v) & =0, \\
\mathcal{A}_{1,0} & :=V_{1}^{\delta} x^{2} \ln x \frac{\partial^{2}}{\partial x^{2}}+V_{2}^{\delta} x v \ln x \frac{\partial^{2}}{\partial x \partial v},
\end{aligned}
$$

and $P_{1,1}^{\delta, \epsilon}=\sqrt{\delta \epsilon} P_{1,1}$ satisfies the PDE

$$
\begin{aligned}
\mathcal{L}_{B S} P_{1,1}^{\delta, \epsilon} & =\mathcal{A}_{1,1} P_{0}, \quad t<T, \\
P_{1,1}^{\delta, \epsilon}(T, x, v) & =0, \\
\mathcal{A}_{1,1} & :=V_{1}^{\delta, \epsilon} x \frac{\partial}{\partial x}\left(x^{2} \ln x \frac{\partial^{2}}{\partial x^{2}}\right)+V_{2}^{\delta, \epsilon} x \frac{\partial}{\partial x}\left(x v \ln x \frac{\partial^{2}}{\partial x \partial v}\right)+V_{3}^{\delta, \epsilon} v \frac{\partial}{\partial v}\left(x^{2} \ln x \frac{\partial^{2}}{\partial x^{2}}\right) \\
& +V_{4}^{\delta, \epsilon} v \frac{\partial}{\partial v}\left(x v \ln x \frac{\partial^{2}}{\partial x \partial v}\right)+V_{5}^{\delta, \epsilon} x^{2} \ln x \frac{\partial^{2}}{\partial x^{2}}+V_{6}^{\delta, \epsilon} x v \ln x \frac{\partial^{2}}{\partial x \partial v},
\end{aligned}
$$

where $\quad V_{1}^{\delta}=\sqrt{\delta} \sigma_{x}^{2}\langle f\rangle, \quad V_{2}^{\delta}=\sqrt{\delta} \rho_{x v} \sigma_{x} \sigma_{v}\langle f\rangle, \quad V_{1}^{\delta, \epsilon}=-\sqrt{\delta \epsilon} \rho_{x y} \sigma_{x}^{3} v \sqrt{2}\left\langle\psi^{\prime}\right\rangle$, $V_{2}^{\delta, \epsilon}=-\sqrt{\delta \epsilon} \rho_{x y} \rho_{x v} \sigma_{x}^{2} \sigma_{v} v \sqrt{2}\left\langle\psi^{\prime}\right\rangle, \quad V_{3}^{\delta, \epsilon}=-\sqrt{\delta \epsilon} \rho_{v y} \sigma_{x}^{2} \sigma_{v} v \sqrt{2}\left\langle\psi^{\prime}\right\rangle, \quad V_{4}^{\delta, \epsilon}=$ $-\sqrt{\delta \epsilon} \rho_{x v} \rho_{v y} \sigma_{x} \sigma_{v}^{2} v \sqrt{2}\left\langle\psi^{\prime}\right\rangle, V_{5}^{\delta, \epsilon}=\sqrt{\delta \epsilon} \sigma_{x}^{2} v \sqrt{2} \Lambda\left\langle\psi^{\prime}\right\rangle, V_{6}^{\delta, \epsilon}=\sqrt{\delta \epsilon} \rho_{x v} \sigma_{x} \sigma_{v} v \sqrt{2} \Lambda\left\langle\psi^{\prime}\right\rangle$, and $\psi$ is the solution of the $O D E \mathcal{L}_{0} \psi(y)=f(y)-\langle f\rangle$ and it is described by the indefinite integral

$$
\psi(y)=\frac{1}{v^{2}} \iint_{-\infty}^{y}(f(y)-\langle f\rangle) \frac{\Phi(z)}{\Phi(y)} d z d y,
$$

where $\Phi$ is the usual standard normal distribution function. 
Proof From the PDE (3.14), we have

$$
\begin{aligned}
\frac{1}{\epsilon} \mathcal{L}_{0} P_{1,0} & +\frac{1}{\sqrt{\epsilon}}\left(\mathcal{L}_{0} P_{1,1}+\mathcal{L}_{10} P_{1,0}+\mathcal{L}_{11} P_{0,0}\right)+\left(\mathcal{L}_{0} P_{1,2}+\mathcal{L}_{10} P_{1,1}+\mathcal{L}_{B S} P_{1,0}+\mathcal{L}_{21} P_{0,0}\right) \\
& +\sqrt{\epsilon}\left(\mathcal{L}_{0} P_{1,3}+\mathcal{L}_{10} P_{1,2}+\mathcal{L}_{B S} P_{1,1}\right)+\cdots=0 .
\end{aligned}
$$

From the order $\frac{1}{\epsilon}$ term of (3.23), we have $\mathcal{L}_{0} P_{1,0}=0$. From the assumed growth condition, $P_{1,0}$ is $y$-independent. Since all the terms in $\mathcal{L}_{10}$ and $\mathcal{L}_{11}$ contain a partial derivative with respect to $y$, we have $\mathcal{L}_{0} P_{1,1}=0$ from the order $\frac{1}{\sqrt{\epsilon}}$ terms of (3.23) and thus $P_{1,1}$ is $y$-independent. This yields $\mathcal{L}_{10} P_{1,1}=0$. Next, from the order 1 terms of (3.23), we have the PDE

$$
\mathcal{L}_{0} P_{1,2}+\mathcal{L}_{B S} P_{1,0}+\mathcal{L}_{21} P_{0,0}=0 .
$$

Since this is a Poisson's equation in $y$ in terms of $\mathcal{L}_{0}$, the Fredholm alternative leads to

$$
\mathcal{L}_{B S} P_{1,0}+\left\langle\mathcal{L}_{21} P_{0,0}\right\rangle=0 .
$$

If we subtract (3.25) from $\mathcal{L}_{B S} P_{1,0}+\mathcal{L}_{21} P_{0,0}$, we obtain

$$
\mathcal{L}_{B S} P_{1,0}+\mathcal{L}_{21} P_{0,0}=\mathcal{L}_{21} P_{0,0}-\left\langle\mathcal{L}_{21} P_{0,0}\right\rangle=\left(\mathcal{L}_{21}-\left\langle\mathcal{L}_{21}\right\rangle\right) P_{0,0} .
$$

By utilizing the definition of $\mathcal{L}_{21}$, we have the desired PDE described by (3.21).

From the order $\sqrt{\epsilon}$ terms of (3.23), we have $\mathcal{L}_{0} P_{1,3}+\mathcal{L}_{10} P_{1,2}+\mathcal{L}_{B S} P_{1,1}=0$. Noticing that this is a Poisson's equation with respect to $\mathcal{L}_{0}$, we obtain

$$
\mathcal{L}_{B S} P_{1,1}=-\left\langle\mathcal{L}_{10} P_{1,2}\right\rangle .
$$

Meanwhile, from (3.24) and (3.26), we have $P_{1,2}$ in the following form

$$
\begin{aligned}
P_{1,2} & =-\mathcal{L}_{0}^{-1}\left(\mathcal{L}_{B S} P_{1,0}+\mathcal{L}_{21} P_{0,0}\right) \\
& =\mathcal{L}_{0}^{-1}\left(\mathcal{L}_{21}-\left\langle\mathcal{L}_{21}\right\rangle\right) P_{0,0} \\
& =-\mathcal{L}_{0}^{-1}(f-\langle f\rangle)\left(\sigma_{x}^{2} x^{2} \ln x \frac{\partial^{2} P_{0,0}}{\partial x^{2}}+\rho_{x v} \sigma_{x} \sigma_{v} x v \ln x \frac{\partial^{2} P_{0,0}}{\partial x \partial v}\right) \\
& =(\psi(y)+c(t, x, v))\left(\sigma_{x}^{2} x^{2} \ln x \frac{\partial^{2} P_{0,0}}{\partial x^{2}}+\rho_{x v} \sigma_{x} \sigma_{v} x v \ln x \frac{\partial^{2} P_{0,0}}{\partial x \partial v}\right)
\end{aligned}
$$

for some function $c(t, x, v)$ which is independent of $y$. Then, by substituting (3.28) into (3.27) and using the definition of the operator $\mathcal{L}_{10}$, we obtain the desired result given by (3.22).

By the same technique as above, $P_{2,0}^{\delta}=\delta P_{2,0}$ satisfies the PDE 


$$
\begin{aligned}
\mathcal{L}_{B S} P_{2,0}^{\delta} & =\mathcal{A}_{1,0} P_{1,0}+\mathcal{A}_{2,0} P_{0}, \\
P_{2,0}^{\delta}(T, x, v) & =0 \\
\mathcal{A}_{2,0} & :=U_{1}^{\delta}(x \ln x)^{2} \frac{\partial^{2}}{\partial x^{2}}+U_{2}^{\delta} x v(\ln x)^{2} \frac{\partial^{2}}{\partial x \partial v},
\end{aligned}
$$

where $U_{1}^{\delta}=-\delta \sigma_{x}^{2}\left\langle f^{2}\right\rangle$ and $U_{2}^{\delta}=-\frac{1}{2} \delta \rho_{x v} \sigma_{x} \sigma_{v}\left\langle f^{2}\right\rangle$.

From Propositions 3.1 and 3.2, and the above result for $P_{2,0}^{\delta}$, the option price $P^{\delta, \epsilon}$ is approximated by

$$
\tilde{P}^{\delta, \epsilon}(t, x, v)=P_{0,0}(t, x, v)+\sqrt{\delta} P_{1,0}(t, x, v)+\sqrt{\delta \epsilon} P_{1,1}(t, x, v)+\delta P_{2,0}(t, x, v) .
$$

The accuracy of this approximation is theoretically given in Sect. 4. The closed form solution of $P_{0,0}$ is given by (3.17) and the closed form solutions of $P_{1,0}^{\delta}, P_{1,1}^{\delta, \epsilon}$ and $P_{2,0}^{\delta}$ will be derived in Sect. 4. Mellin transform is a powerful tool to derive the analytic solutions.

\section{Mellin Transform for Option Pricing}

To derive analytic solutions for $P_{1,0}^{\delta}, P_{1,1}^{\delta, \epsilon}$ and $P_{2,0}^{\delta}$, we use the double Mellin transform method in this section. A brief review of it is as follows. For a locally Lebesgue integrable function $f(x, v),(x, v) \in \mathbb{R}_{+}^{2}$, the double Mellin transform $\mathcal{M}\left(f(x, v), x^{*}, v^{*}\right),\left(x^{*}, v^{*}\right) \in \mathbb{C}^{2}$ is defined by

$$
\mathcal{M}\left(f(x, v), x^{*}, v^{*}\right):=\hat{f}\left(x^{*}, v^{*}\right)=\int_{0}^{\infty} \int_{0}^{\infty} f(x, v) x^{x^{*}-1} v^{v^{*}-1} d x d v,
$$

and if $a<\operatorname{Re}\left(x^{*}\right), \operatorname{Re}\left(v^{*}\right)<b$ and $c_{1}$ and $c_{2}$ are given such that $a<c_{1}, c_{2}<b$, then the inverse of the double Mellin transform is expressed as

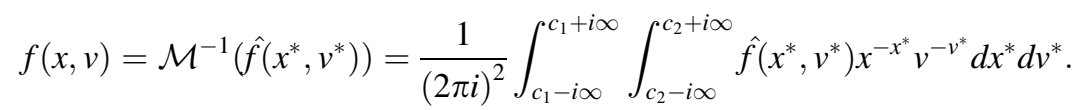

The leading term $P_{0,0}$ is the European vulnerable option price under the BlackScholes model with constant volatility. The existence of the double Mellin transform of $P_{0,0}$ was discussed in Yoon and Kim (2015) and it was explicitly derived by Yoon et al. (2013). Let $\widehat{P}_{0}\left(t, x^{*}, v^{*}\right), \widehat{P}_{1,0}^{\delta}\left(t, x^{*}, v^{*}\right), \widehat{P}_{1,1}^{\delta, \epsilon}\left(t, x^{*}, v^{*}\right)$ and $\widehat{P}_{2,0}^{\delta}\left(t, x^{*}, v^{*}\right)$ be the double Mellin transforms of $P_{0,0}(t, x, v), P_{1,0}^{\delta}(t, x, v), P_{1,1}^{\delta, \epsilon}(t, x, v)$ and $P_{2,0}^{\delta}(t, x, v)$, respectively. Then, from the properties of the double Mellin transform, the PDEs (3.21), (3.22) and (3.29) are transformed into ODEs given by 


$$
\begin{aligned}
& \frac{\partial \widehat{P}_{1,0}^{\delta}}{\partial t}+A\left(x^{*}, v^{*}\right) \widehat{P}_{1,0}^{\delta}=\frac{\partial}{\partial x^{*}}\left(q_{1}\left(x^{*}, v^{*}\right) \widehat{P}_{0}\right), \\
& A\left(x^{*}, v^{*}\right):=\frac{\sigma_{x}^{2}}{2} x^{* 2}+\frac{\sigma_{v}^{2}}{2} v^{* 2}+\rho_{x v} \sigma_{x} \sigma_{v} x^{*} v^{*}-\left(r-\frac{\sigma_{x}^{2}}{2}\right) x^{*}-\left(r-\frac{\sigma_{v}^{2}}{2}\right) v^{*}-r, \\
& q_{1}\left(x^{*}, v^{*}\right):=V_{1}^{\delta}\left(x^{*}+x^{* 2}\right)+V_{2}^{\delta} v^{*} x^{*}, \\
& \frac{\partial \widehat{P}_{1,1}^{\delta, \epsilon}}{\partial t}+A\left(x^{*}, v^{*}\right) \widehat{P}_{1,1}^{\delta}=q_{2}\left(x^{*}, v^{*}\right) \widehat{P}_{0}+\frac{\partial}{\partial x^{*}}\left(q_{3}\left(x^{*}, v^{*}\right) \widehat{P}_{0}\right), \\
& q_{2}\left(x^{*}, v^{*}\right):=V_{1}^{\delta, \epsilon}\left(x^{*}+x^{* 2}\right)+V_{2}^{\delta, \epsilon} v^{*} x^{*} \\
& q_{3}\left(x^{*}, v^{*}\right):=-V_{1}^{\delta, \epsilon} x^{*}\left(x^{*}+x^{* 2}\right)-V_{2}^{\delta, \epsilon} v^{*} x^{* 2}-V_{3}^{\delta, \epsilon} v^{*}\left(x^{*}+x^{* 2}\right) \\
& -V_{4}^{\delta, \epsilon} x^{*} v^{* 2}+V_{5}^{\delta, \epsilon}\left(x^{*}+x^{* 2}\right)+V_{6}^{\delta, \epsilon} v^{*} x^{*}
\end{aligned}
$$

and

$$
\begin{gathered}
\frac{\partial \widehat{P}_{2,0}^{\delta}}{\partial t}+A\left(x^{*}, v^{*}\right) \widehat{P}_{2,0}^{\delta}=\frac{\partial}{\partial x^{*}}\left(q_{1}\left(x^{*}, v^{*}\right) \widehat{P}_{1,0}\right)+\frac{\partial^{2}}{\partial x^{* 2}}\left(q_{4}\left(x^{*}, v^{*}\right) \widehat{P}_{0}\right), \\
q_{4}\left(x^{*}, v^{*}\right):=U_{1}^{\delta}\left(x^{*}+x^{* 2}\right)+U_{2}^{\delta} v^{*} x^{*}
\end{gathered}
$$

respectively.

Next, we solve these ODEs. We first prove the following lemma first.

Lemma 4.1 The solution of the $O D E$

$$
\frac{\partial \widehat{P}}{\partial t}+A\left(x^{*}, v^{*}\right) \widehat{P}=\tilde{q}_{1}\left(x^{*}, v^{*}\right) \widehat{P}_{0}+\frac{\partial}{\partial x^{*}}\left(\tilde{q}_{2}\left(x^{*}, v^{*}\right) \widehat{P}_{0}\right)
$$

is given by

$$
\begin{aligned}
\widehat{P}\left(t, x^{*}, v^{*}\right)= & (t-T)\left(\tilde{q}_{1}\left(x^{*}, v^{*}\right) \widehat{P}_{0}+\frac{\partial}{\partial x^{*}}\left(\tilde{q}_{2}\left(x^{*}, v^{*}\right) \widehat{P}_{0}\right)\right) \\
& +\frac{1}{2}(t-T)^{2} \frac{\partial A}{\partial x^{*}} \tilde{q}_{2}\left(x^{*}, v^{*}\right) \widehat{P}_{0},
\end{aligned}
$$

where $\widehat{P}\left(t, x^{*}, v^{*}\right)$ is the double Mellin transform of $P(t, x, v)$ and $\tilde{q}_{1}$ and $\tilde{q}_{2}$ are some functions of $x^{*}$ and $v^{*}$.

Proof We seek a solution of (4.9) in the form

$$
\widehat{P}\left(t, x^{*}, v^{*}\right)=\alpha\left(t, x^{*}, v^{*}\right) \widehat{P}_{0}+\beta\left(t, x^{*}, v^{*}\right) \frac{\partial}{\partial x^{*}}\left(\tilde{q}_{2}\left(x^{*}, v^{*}\right) \widehat{P}_{0}\right) .
$$


Plugging this into (4.9) yields

$$
\begin{aligned}
\frac{\partial \alpha}{\partial t}\left(t, x^{*}, v^{*}\right) & =\beta \frac{\partial A}{\partial x^{*}} \tilde{q}_{2}+\tilde{q}_{1}, \\
\alpha\left(T, x^{*}, v^{*}\right) & =0, \\
\frac{\partial \beta}{\partial t}\left(t, x^{*}, v^{*}\right) & =1, \\
\beta\left(T, x^{*}, v^{*}\right) & =0 .
\end{aligned}
$$

We can easily solve these equations whose solutions are given by

$$
\begin{aligned}
& \alpha\left(t, x^{*}, v^{*}\right)=\frac{1}{2}(t-T)^{2} \frac{\partial A}{\partial x^{*}} \tilde{q}_{2}+(t-T) \tilde{q}_{1}, \\
& \beta\left(t, x^{*}, v^{*}\right)=t-T .
\end{aligned}
$$

Therefore, we obtain the solution of $\widehat{P}\left(t, x^{*}, v^{*}\right)$ given by (4.10).

By making use of Lemma 4.1 and (4.1) and (4.4), we obtain

$$
\begin{gathered}
\widehat{P}_{1,0}^{\delta}\left(t, x^{*}, v^{*}\right)=(t-T) \frac{\partial}{\partial x^{*}}\left(q_{1} \widehat{P}_{0}\right)+\frac{1}{2}(t-T)^{2} \frac{\partial A}{\partial x^{*}} q_{1} \widehat{P}_{0}, \\
\widehat{P}_{1,1}^{\delta, \epsilon}\left(t, x^{*}, v^{*}\right)=(t-T)\left(q_{2} \widehat{P}_{0}+\frac{\partial}{\partial x^{*}}\left(q_{3} \widehat{P}_{0}\right)\right)+\frac{1}{2}(t-T)^{2} \frac{\partial A}{\partial x^{*}} q_{3} \widehat{P}_{0},
\end{gathered}
$$

where $q_{1}, q_{2}$ and $q_{3}$ are given by (4.3), (4.5) and (4.6), respectively. So, we obtain the following results.

Proposition 4.1 The correction terms $P_{1,0}^{\delta}$ and $P_{1,1}^{\delta, \epsilon}$ are given by

$$
\begin{gathered}
P_{1,0}^{\delta}(t, x, v)=(t-T) \mathcal{A}_{1,0} P_{0}+\frac{1}{2}(t-T)^{2} \mathcal{Q}_{A} \mathcal{Q}_{1} P_{0}, \\
P_{1,1}^{\delta, \epsilon}(t, x, v)=(t-T) \mathcal{A}_{1,1} P_{0}+\frac{1}{2}(t-T)^{2} \mathcal{Q}_{A} \mathcal{Q}_{3} P_{0} \\
\mathcal{Q}_{A}:=\left(\frac{\sigma_{x}^{2}}{2}-r\right) \cdot-\sigma_{x}^{2} x \frac{\partial}{\partial x}-\rho_{x v} \sigma_{x} \sigma_{v} v \frac{\partial}{\partial v}, \\
\mathcal{Q}_{3}:=V_{1}^{\delta, \epsilon} x \frac{\partial}{\partial x}\left(x^{2} \frac{\partial^{2}}{\partial x^{2}}\right)+V_{2}^{\delta} \frac{\partial^{2}}{\partial x^{2}}+V_{2}^{\delta} x v \frac{\partial}{\partial v}\left(x \frac{\partial}{\partial x}\right)^{2}, \\
+V_{3}^{\delta, \epsilon} v \frac{\partial}{\partial v}\left(x^{2} \frac{\partial^{2}}{\partial x^{2}}\right)+V_{4}^{\delta, \epsilon} x \frac{\partial}{\partial x}\left(v \frac{\partial}{\partial v}\right)^{2}+V_{5}^{\delta, \epsilon} x^{2} \frac{\partial^{2}}{\partial x^{2}}+V_{6}^{\delta, \epsilon} x v \frac{\partial^{2}}{\partial x \partial v}
\end{gathered}
$$

respectively, where $\mathcal{A}_{1,0}$ and $\mathcal{A}_{1,1}$ are defined by (3.21) and (3.22), respectively. 
Proof From the definition of $A\left(x^{*}, v^{*}\right)$ in (4.2), we have

$$
\frac{\partial A}{\partial x^{*}}=\left(\frac{\sigma_{x}^{2}}{2}-r\right)+\sigma_{x}^{2} x^{*}+\rho_{x v} \sigma_{x} \sigma_{v} v^{*}
$$

Applying the inverse double Mellin transform on $\widehat{P}_{1,0}^{\delta}$ in (4.12) and making use of the properties of the inverse of double Mellin transform yield

$$
\begin{aligned}
P_{1,0}^{\delta}(t, x, v) & =\mathcal{M}^{-1}\left(\widehat{P}_{1,0}^{\delta}\left(t, x^{*}, v^{*}\right)\right) \\
& =\mathcal{M}^{-1}\left((t-T) \frac{\partial}{\partial x^{*}}\left(q_{1} \widehat{P}_{0}\right)+\frac{1}{2}(t-T)^{2} \frac{\partial A}{\partial x^{*}} q_{1} \widehat{P}_{0}\right) \\
& =(t-T) \mathcal{M}^{-1}\left(\frac{\partial}{\partial x^{*}}\left(q_{1} \widehat{P}_{0}\right)\right)+\frac{1}{2}(t-T)^{2} \mathcal{M}^{-1}\left(\frac{\partial A}{\partial x^{*}} q_{1} \widehat{P}_{0}\right) \\
& =(t-T) \mathcal{A}_{1,0} P_{0}+\frac{1}{2}(t-T)^{2} \mathcal{Q}_{A} \mathcal{Q}_{1} P_{0},
\end{aligned}
$$

where $\mathcal{Q}_{A}, \mathcal{Q}_{1}$ and $\mathcal{A}_{1,0}$ are defined as (4.16), (4.17) and (3.21), respectively.

Similarly, to obtain the explicit expression for $P_{1,1}^{\delta, \epsilon}$, we apply the inverse double Mellin transform to (4.13) which gives

$$
\begin{aligned}
P_{1,1}^{\delta, \epsilon}(t, x, v) & =\mathcal{M}^{-1}\left(\widehat{P}_{1,1}^{\delta, \epsilon}\left(t, x^{*}, v^{*}\right)\right) \\
& =\mathcal{M}^{-1}\left((t-T)\left(q_{2} \widehat{P}_{0}+\frac{\partial}{\partial x^{*}}\left(q_{3} \widehat{P}_{0}\right)\right)+\frac{1}{2}(t-T)^{2} \frac{\partial A}{\partial x^{*}} q_{3} \widehat{P}_{0}\right) \\
& =(t-T) \mathcal{M}^{-1}\left(\frac{\partial}{\partial x^{*}}\left(q_{2} \widehat{P}_{0}+q_{3} \widehat{P}_{0}\right)\right)+\frac{1}{2}(t-T)^{2} \mathcal{M}^{-1}\left(\frac{\partial A}{\partial x^{*}} q_{3} \widehat{P}_{0}\right) \\
& =(t-T) \mathcal{A}_{1,1} P_{0}+\frac{1}{2}(t-T)^{2} \mathcal{Q}_{A} \mathcal{Q}_{3} P_{0},
\end{aligned}
$$

where $\mathcal{Q}_{A}, \mathcal{Q}_{3}$ and $\mathcal{A}_{1,1}$ are defined as (4.16), (4.18) and (3.22), respectively.

The following lemma gives the solution of (4.7).

Lemma 4.2 The solution of the ODE (4.7), i.e.,

$$
\frac{\partial \widehat{P}_{2,0}^{\delta}}{\partial t}+A\left(x^{*}, v^{*}\right) \widehat{P}_{2,0}^{\delta}=\frac{\partial}{\partial x^{*}}\left(q_{1}\left(x^{*}, v^{*}\right) \widehat{P}_{1,0}\right)+\frac{\partial^{2}}{\partial x^{* 2}}\left(q_{4}\left(x^{*}, v^{*}\right) \widehat{P}_{0}\right)
$$

is given by

$$
\begin{aligned}
\widehat{P}_{2,0}^{\delta}\left(t, x^{*}, v^{*}\right)= & (t-T)\left(\frac{\partial}{\partial x^{*}}\left(q_{1} \widehat{P}_{1,0}\right)+\frac{\partial^{2}}{\partial x^{* 2}}\left(q_{4} \widehat{P}_{0}\right)\right)+\alpha_{1}\left(t, x^{*}, v^{*}\right) \widehat{P}_{0} \\
& +\beta_{1}\left(t, x^{*}, v^{*}\right) \frac{\partial \widehat{P}_{0}}{\partial x^{*}}+\gamma_{1}\left(t, x^{*}, v^{*}\right) \frac{\partial^{2} \widehat{P}_{0}}{\partial x^{* 2}},
\end{aligned}
$$


where

$$
\begin{gathered}
\alpha_{1}\left(t, x^{*}, v^{*}\right)=\frac{1}{8}(t-T)^{4} q_{1}^{2}\left(\frac{\partial A}{\partial x^{*}}\right)^{2}+(t-T)^{3}\left(\frac{\partial A}{\partial x^{*}}\left(-\frac{1}{6} q_{1} \frac{\partial q_{1}}{\partial x^{*}}+\frac{1}{3} q_{4} \frac{\partial A}{\partial x^{*}}\right)-\frac{1}{6} \sigma_{x}^{2} q_{1}^{2}\right) \\
+(t-T)^{2}\left(\frac{1}{2} \sigma_{x}^{2} q_{4}+\frac{\partial A}{\partial x^{*}} \frac{\partial q_{4}}{\partial x^{*}}-\frac{1}{2}\left(\frac{\partial q_{1}}{\partial x^{*}}\right)^{2}-V_{1}^{\delta} q_{1}\right) \\
\beta_{1}\left(t, x^{*}, v^{*}\right)=(t-T)^{2}\left(-\frac{3}{2} q_{1} \frac{\partial q_{1}}{\partial x^{*}}+q_{4} \frac{\partial A}{\partial x^{*}}\right) \\
\gamma_{1}\left(t, x^{*}, v^{*}\right)=-\frac{1}{2}(t-T)^{2} q_{1}^{2}
\end{gathered}
$$

Proof We seek a solution of (4.20) in the form $\widehat{P}_{2,0}^{\delta}\left(t, x^{*}, v^{*}\right)=(t-T)\left(\frac{\partial}{\partial x^{*}}\left(q_{1} \widehat{P}_{1,0}\right)+\frac{\partial^{2}}{\partial x^{* 2}}\left(q_{4} \widehat{P}_{0}\right)\right)+\alpha_{1}\left(t, x^{*}, v^{*}\right) \widehat{P}_{0}+\beta_{1}\left(t, x^{*}, v^{*}\right) \frac{\partial \widehat{P}_{0}}{\partial x^{*}}+\gamma_{1}\left(t, x^{*}, v^{*}\right) \frac{\partial^{2} \widehat{P}_{0}}{\partial x^{* 2}}$.

Substituting this into (4.20) yields the following ODEs for $\alpha_{1}, \beta_{1}$ and $\gamma_{1}$.

$$
\begin{aligned}
\frac{\partial \alpha_{1}}{\partial t}\left(t, x^{*}, v^{*}\right)= & -(t-T)\left(\left(\frac{\partial q_{1}}{\partial x^{*}}\right)^{2}+2 V_{1}^{\delta} q_{1}-\frac{\partial A}{\partial x^{*}} q_{1}\left(\frac{1}{2}(t-T)^{2} \frac{\partial A}{\partial x^{*}} q_{1}+(t-T) \frac{\partial q_{1}}{\partial x^{*}}\right)\right) \\
& (t-T)\left(q_{4} \frac{\partial^{2} A}{\partial x^{* 2}}+2 \frac{\partial A}{\partial x^{*}} \frac{\partial q_{4}}{\partial x^{*}}\right)+\beta_{1} \frac{\partial A}{\partial x^{*}}+\gamma_{1} \frac{\partial^{2} A}{\partial x^{* 2}}, \\
\alpha_{1}\left(T, x^{*}, v^{*}\right)= & 0, \\
\frac{\partial \beta_{1}}{\partial t}\left(t, x^{*}, v^{*}\right)= & -(t-T)\left(3 q_{1} \frac{\partial q_{1}}{\partial x^{*}}-(t-T) \frac{\partial A}{\partial x^{*}} q_{1}^{2}\right)+2(t-T) q_{4} \frac{\partial A}{\partial x^{*}}+2 \gamma_{1} \frac{\partial A}{\partial x^{*}}, \\
\beta_{1}\left(T, x^{*}, v^{*}\right)= & 0, \\
\frac{\partial \gamma_{1}}{\partial t}\left(t, x^{*}, v^{*}\right)= & -(t-T) q_{1}^{2}, \\
\gamma_{1}\left(T, x^{*}, v^{*}\right)= & 0 .
\end{aligned}
$$

One can easily solve these equations and they are given by (4.22), (4.23) and (4.24), respectively.

Using Lemma 4.2, the inverse double Mellin transform and its properties, we obtain 


$$
\begin{aligned}
P_{2,0}^{\delta}(t, x, v)= & \mathcal{M}^{-1}\left(\widehat{P}_{2,0}^{\delta}\left(t, x^{*}, v^{*}\right)\right) \\
= & \mathcal{M}^{-1}\left((t-T)\left(\frac{\partial}{\partial x^{*}}\left(q_{1} \widehat{P}_{1,0}\right)+\frac{\partial^{2}}{\partial x^{* 2}}\left(q_{4} \widehat{P}_{0}\right)\right)\right. \\
& \left.+\alpha_{1}\left(t, x^{*}, v^{*}\right) \widehat{P}_{0}+\beta_{1}\left(t, x^{*}, v^{*}\right) \frac{\partial \widehat{P}_{0}}{\partial x^{*}}+\gamma_{1}\left(t, x^{*}, v^{*}\right) \frac{\partial^{2} \widehat{P}_{0}}{\partial x^{* 2}}\right) \\
= & (t-T) \mathcal{M}^{-1}\left(\frac{\partial}{\partial x^{*}}\left(q_{1} \widehat{P}_{1,0}\right)+\frac{\partial^{2}}{\partial x^{* 2}}\left(q_{4} \widehat{P}_{0}\right)\right) \\
& +\mathcal{M}^{-1}\left(\alpha_{1}\left(t, x^{*}, v^{*}\right) \widehat{P}_{0}+\beta_{1}\left(t, x^{*}, v^{*}\right) \frac{\partial \widehat{P}_{0}}{\partial x^{*}}+\gamma_{1}\left(t, x^{*}, v^{*}\right) \frac{\partial^{2} \widehat{P}_{0}}{\partial x^{* 2}}\right) \\
= & (t-T)\left(\mathcal{A}_{1,0} P_{1,0}+\mathcal{A}_{2,0} P_{0}\right) \\
& +\mathcal{M}^{-1}\left(\alpha_{1}\left(t, x^{*}, v^{*}\right) \widehat{P}_{0}+\beta_{1}\left(t, x^{*}, v^{*}\right) \frac{\partial \widehat{P}_{0}}{\partial x^{*}}+\gamma_{1}\left(t, x^{*}, v^{*}\right) \frac{\partial^{2} \widehat{P}_{0}}{\partial x^{* 2}}\right),
\end{aligned}
$$

where $\mathcal{A}_{1,0}$ and $\mathcal{A}_{2,0}$ are given by (3.21) and (3.29), respectively. Then, by using explicit expression for $P_{1,0}^{\delta}$ given by (4.14), direct computation gives

$$
\begin{gathered}
P_{2,0}^{\delta}(t, x, v)=(t-T)(\ln x)^{2} \mathcal{Q}_{4} P_{0}+\frac{1}{2}(t-T)^{2}(\ln x)^{2} \mathcal{Q}_{1}^{2} P_{0}+(t-T)^{2} \\
\ln x\left(\mathcal{Q}_{4} \mathcal{Q}_{A}-\frac{1}{2} \mathcal{Q}_{1} \mathcal{Q}_{2}\right) P_{0} \\
-\frac{1}{2}(t-T)^{2} \sigma_{x}^{2} \mathcal{Q}_{4} P_{0}+\frac{1}{2}(t-T)^{3} \ln x \mathcal{Q}_{A} \mathcal{Q}_{1}^{2} P_{0} \\
+(t-T)^{3}\left(\frac{1}{3} \mathcal{Q}_{A}\left(\mathcal{Q}_{4} \mathcal{Q}_{A}-\frac{1}{2} \mathcal{Q}_{1} \mathcal{Q}_{2}\right)-\frac{1}{6} \sigma_{x}^{2} \mathcal{Q}_{1}^{2}\right) P_{0}+\frac{1}{8}(t-T)^{4}\left(\mathcal{Q}_{1} \mathcal{Q}_{A}\right)^{2} P_{0} \\
\mathcal{Q}_{2}:=V_{1}^{\delta}\left(\cdot-2 x \frac{\partial}{\partial x}\right)-V_{2}^{\delta} v \frac{\partial}{\partial v} \\
\mathcal{Q}_{4}:=U_{1}^{\delta} x^{2} \frac{\partial^{2}}{\partial x^{2}}+U_{2}^{\delta} x v \frac{\partial^{2}}{\partial x \partial v} .
\end{gathered}
$$

To sum up, we have derived the corresponding ODEs from the pricing PDEs through the utilization of Mellin transform and then found the solutions of the ODEs based upon Lemmas 4.1 and 4.2. Finally, the pricing correction terms $P_{1,0}^{\delta}(t, x, v), P_{1,1}^{\delta, \epsilon}(t, x, v)$ and $P_{2,0}^{\delta}(t, x, v)$ are obtained by the inverse double Mellin transform and its properties.

Proposition 4.2 Let $P^{\delta, \epsilon}(t, x, y, v)$ be the solution of the PDE problem (2.8) and $\tilde{P}^{\delta, \epsilon}(t, x, v)$ an approximation given by (3.30). When the payoff function $h(x, v)$ is continuous, for any $(t, x, y, z)$ and for any $0<\epsilon \leq 1$ and $0<\delta \leq 1$, we have 


$$
\left|P(t, x, y, v)-\tilde{P}^{\delta, \epsilon}(t, x, v)\right|=\mathcal{O}\left(\epsilon^{3 / 2-}+\epsilon \sqrt{\delta}+\delta^{3 / 2}\right),
$$

where notation $\mathcal{O}\left(\epsilon^{3 / 2-}\right)$ represents terms that are of order $\mathcal{O}\left(\epsilon^{1+q / 2}\right)$ for any $q<1$.

Proof The proof of this proposition is similar to the proof of the Proposition 2.5 in Fouque et al. (2016) and the proof of Theorem 4.1 in Choi et al. (2016). In particular, Fouque et al. (2016) provides the detailed explanation for the second order approximation of the option price so that readers can understand the error accuracy mentioned in (4.28). In addition, compared to Choi et al. (2016), the only difference of our paper is that the payoff function $h$ depends on an additional process $V_{t}$ in this paper. Since the payoff function of vulnerable option is bounded by the payoff function of European vanilla option, that is, $h\left(X_{t}, V_{t}\right) \leq\left(X_{t}-K\right)^{+}$, the proof of accuracy does not deviate from that of Choi et al. (2016). Therefore, combining the above two references, the verification for the error estimation in (4.28) is completed.

We present a numerical evidence of the accuracy (4.28) by computing and comparing the approximation and the exact solution in the following section.

\section{Implications}

This section shows the price behavior of a vulnerable call option with respect to the model parameters as well as the price accuracy of our analytic formula. In the previous section, we have shown that the option price is expressed as an explicit closed form by using the Mellin transform techniques. Due to the analytical solution, we are able to analyze the sensitivity of the option price against the model parameters in an effective manner.

Figure 1 exhibits the price behavior of a vulnerable ATM call option against the underlying asset price $(x)$ and the firm value $(v)$ of the option writer $(\mathrm{OW})$ assets for different choices of the volatilities $\left(\sigma_{x}\right.$ and $\sigma_{v}$ ) of the asset and the firm value. We choose the same values of the two volatilities because we believe that they go along with each other in general. As shown in the figure, when the underlying asset price, the firm value of the option writer, or the volatilities increases, the price of the vulnerable call option rises as it should be the case. A noticeable fact is that the value of the vulnerable call option falls more sharply when the firm value is smaller than the critical value $D^{*}$ as the volatilities become smaller. It implies that an event of default is more shocking to the call option buyer when the market has been less volatile.

Next, we investigate the analytical option price accuracy numerically through the Monte Carlo (MC) simulation. Table 1 displays the option price $\tilde{P}^{\delta, \epsilon}$ given by (3.30) compared to the $\mathrm{MC}$ result denoted by $P_{\mathrm{MC}}$. The $\mathrm{MC}$ option price $P_{\mathrm{MC}}$ is calculated with 100,000 simulation paths, where the parameter values are $x=100, y=$ $0.3, v=100, r=0.03, \sigma_{x}=0.2, \sigma_{v}=0.3, \sigma_{y}=0.5, v=0.01, \rho_{x y}=-0.1, \quad \rho_{x v}=$ $0.2, \rho_{y v}=-0.2, K=100, D^{*}=85, \alpha=0.5, T=1, f(y)=e^{-y},\langle f\rangle=0.01,\left\langle\psi^{\prime}\right\rangle=$ 


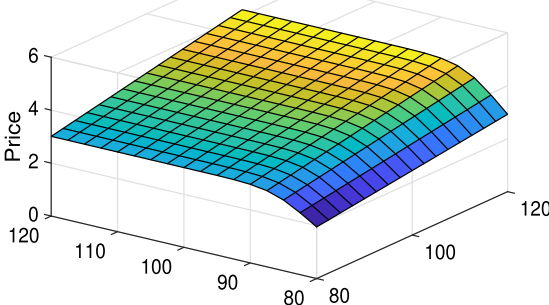

Firm value of the option writer Underlying asset price

(a) $\sigma_{x}=\sigma_{v}=0.05$

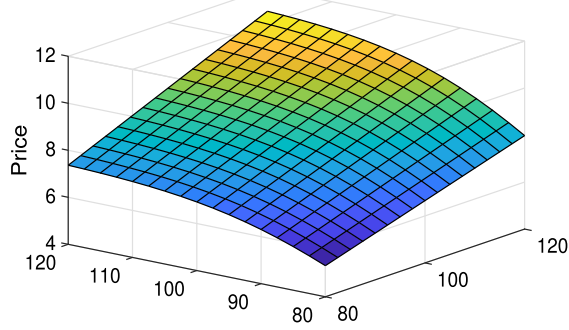

Firm value of the option writer

Underlvina asset price

(c) $\sigma_{x}=\sigma_{v}=0.2$

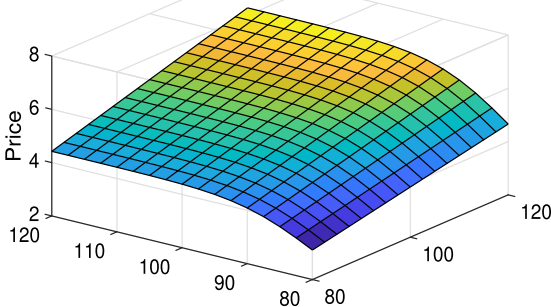

Firm value of the option writer

Underlying asset price

(b) $\sigma_{x}=\sigma_{v}=0.1$

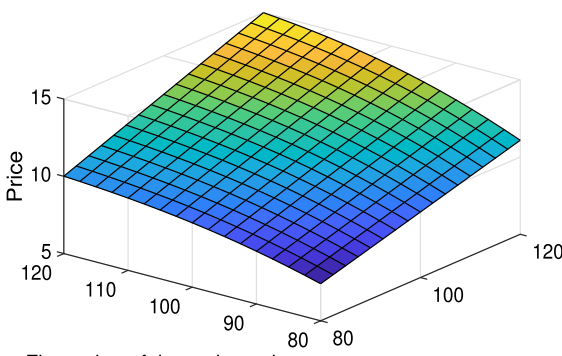

Firm value of the option writer

Underlying asset price

(d) $\sigma_{x}=\sigma_{v}=0.3$

Fig. 1 Value of a vulnerable ATM call option against the firm value of the option writer and the underlying asset price

Table 1 Comparison between the analytical solution $\tilde{P}^{\delta, \epsilon}$ and the numerical solution $P_{\mathrm{MC}}$, where "Error" means the relative percentage error defined by $\left|\frac{P_{\mathrm{MC}}-\tilde{P}^{\tilde{\delta}, \epsilon}}{P_{\mathrm{MC}}}\right| \times 100$

\begin{tabular}{lllllll}
\hline & \multicolumn{5}{l}{$\tilde{P}^{\delta, \epsilon}$} & \multicolumn{3}{c}{} & \multirow{2}{*}{$P_{\mathrm{MC}}$} \\
\cline { 2 - 6 } & $\delta=1$ & $\delta=0.5$ & $\delta=0.1$ & $\delta=0.05$ & $\delta=0.01$ & \\
\hline$\epsilon=1$ & 7.8403 & 7.9429 & 8.0812 & 8.1142 & 8.1584 & 8.1659 \\
Error(\%) & 3.9862 & 2.7305 & 1.036 & 0.6327 & 0.0914 & - \\
$\epsilon=0.5$ & 7.8538 & 7.9524 & 8.0854 & 8.1172 & 8.1597 & 8.1667 \\
Error(\%) & 3.8310 & 2.6237 & 0.9948 & 0.6059 & 0.0851 & - \\
$\epsilon=0.1$ & 7.8718 & 7.9651 & 8.0911 & 8.1212 & 8.1615 & 8.1680 \\
Error(\%) & 3.6266 & 2.4840 & 0.9414 & 0.5729 & 0.0794 & - \\
$\epsilon=0.05$ & 7.8761 & 7.9681 & 8.0925 & 8.1222 & 8.1620 & 8.1682 \\
Error(\%) & 3.5768 & 2.4495 & 0.9274 & 0.5637 & 0.0767 & - \\
$\epsilon=0.01$ & 7.8817 & 7.9722 & 8.0943 & 8.1234 & 8.1625 & 8.1684 \\
Error(\%) & 3.5091 & 2.4022 & 0.9073 & 0.5502 & 0.0717 & - \\
\hline
\end{tabular}


$-0.1, \Lambda=1$. In addition, we find the relative percentage error given by $\left|\frac{P_{\mathrm{MC}}-\tilde{P} \delta, \epsilon}{P_{\mathrm{MC}}}\right| \times$ 100 in Table 1. As one can notice from the table, the gap between the analytical option price and the numerical price becomes smaller (more rapidly in $\delta$ than $\epsilon$ ) as $\epsilon$ or $\delta$ gets close to zero.

We, now, find the stochastic elasticity of variance (SEV) effect on the vulnerable option price by comparing to the Black-Scholes (BS) price for the vulnerable option. Figure 2 shows differences between the BS option price and the SEV option price against the firm value of the option writer for three different choices of the critical level $D^{*}$ (the critical value of default) and $\alpha$ (the deadweight costs). As seen in the figure, the price difference becomes larger when $D^{*}$ or $\alpha$ decreases. It is the case also when the firm value of the option writer or time-to-maturity increases. If the firm value is large enough near the maturity, then the difference becomes a maximum level and independent of $D^{*}, \alpha$ and the firm value.

Figure 3 exhibits the Greek, the first derivative of the option price with respect to the market price of risk $\Lambda$, given by

$$
\begin{aligned}
\frac{\partial P^{\tilde{\delta}, \epsilon}}{\partial \Lambda}=\frac{\partial P_{1,1}^{\delta, \epsilon}}{\partial \Lambda} & =(t-T)\left\{V_{5}^{* \delta, \epsilon} x^{2} \ln x \frac{\partial^{2} P_{0}}{\partial x^{2}}+V_{6}^{* \delta, \epsilon} x v \ln x \frac{\partial^{2} P_{0}}{\partial x \partial v}\right\} \\
& -\frac{1}{2}(t-T)^{2} \mathcal{Q}_{A}\left\{V_{5}^{* \delta, \epsilon} x^{2} \frac{\partial^{2} P_{0}}{\partial x^{2}}+V_{6}^{* \delta, \epsilon} x v \frac{\partial^{2} P_{0}}{\partial x \partial v}\right\}
\end{aligned}
$$

where $V_{5}^{* \delta, \epsilon}=\frac{\partial V_{5}^{\delta, \epsilon}}{\partial \Lambda}=\sqrt{2 \delta \epsilon} \sigma_{x}^{2} v\left\langle\psi^{\prime}\right\rangle$, and $V_{6}^{* \delta, \epsilon}=\frac{\partial V_{6}^{\delta, \epsilon}}{\partial \Lambda}=\sqrt{2 \delta \epsilon} \rho_{x v} \sigma_{x} \sigma_{v} v\left\langle\psi^{\prime}\right\rangle$, and $\mathcal{Q}_{A}$ is given by (4.15). As shown in the figure, the slope of the Greek $\frac{\partial \tilde{P}}{\partial \Lambda}$ increases when the firm value of the OW decreases. It says that the sensitivity of the Greek is rising as the defaulting probability is increasing. In particular, for shorter time to maturity as in Figure 3-(c), the slope of the Greek increases drastically until the firm value of the OW decreases to $D^{*}$. It implies that the change of market price of risk has a more significant influence on the option price in the case of higher probability of default and shorter time to maturity.

\section{Conclusion}

There are two possible ways of developing stochastic volatility frameworks from the Black-Scholes model. One way is to randomize the constant volatility of the Black-Scholes model. The Heston model is the best example of it. The other method is to randomize the constant elasticity of the Black-Scholes or CEV model. The SEV model used in this paper belong to this category.

The Heston model or Choi et al. (2016) provides semi-analytical pricing formulas for European vanilla options. These formulas require the calculation of logarithms with complex arguments during the process of computing the inverse Fourier integral. Subsequently, this leads to a numerical instability for a certain range of time to maturities or fast mean reversion, which may ultimately come up with inaccurate solutions and reduce the performance of the option's data fitting. In 


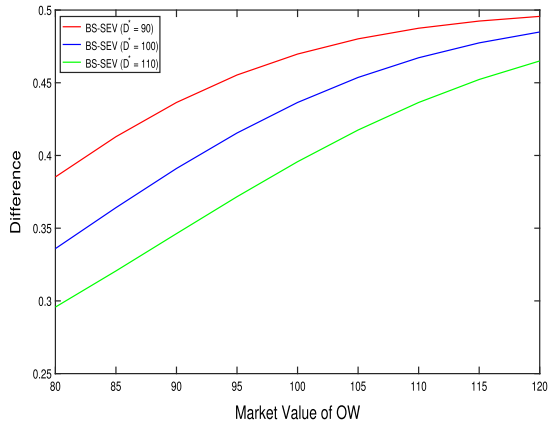

(a) $\alpha=0.2$ and $T=1$

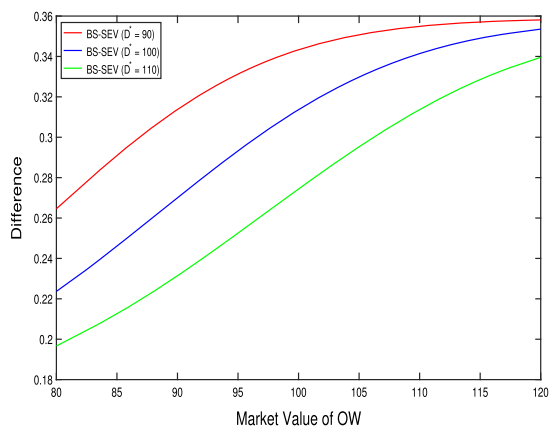

(c) $\alpha=0.2$ and $T=0.5$

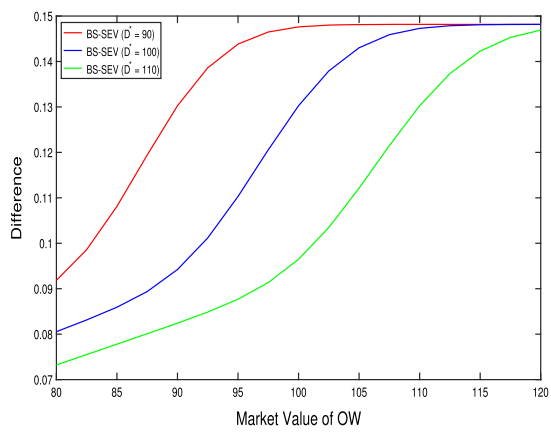

(e) $\alpha=0.2 T=1 / 12$

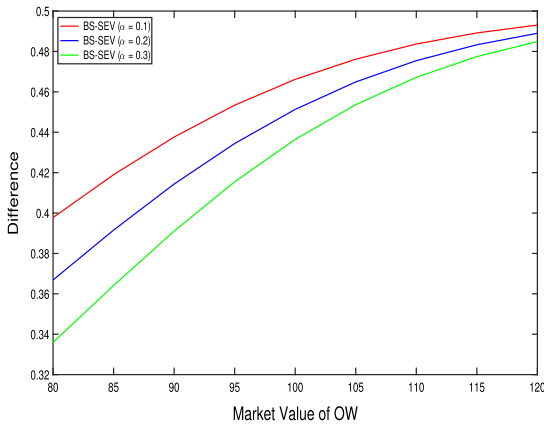

(b) $D^{*}=100$ and $T=1$

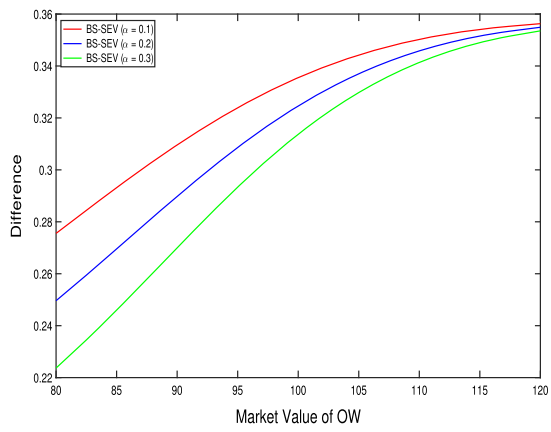

(d) $D^{*}=100$ and $T=0.5$

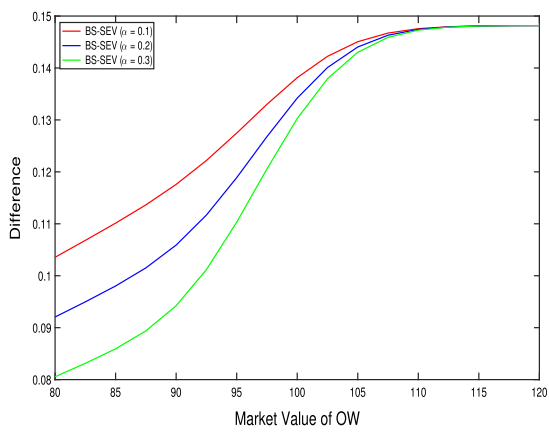

(f) $D^{*}=100$ and $T=1 / 12$

Fig. 2 Difference between the Black-Scholes and the SEV vulnerable call option prices against the firm value of the option writer for three different time-to-maturities

addition, when it comes to solving the solution numerically, it may take much time for us to compute the option price and implement option's data fitting. In this paper, we use the SEV model to obtain a closed form analytical pricing formula for the European options via the Mellin transform approach. This formula only requires the evaluation of the Black-Scholes formula and its derivatives. So, the SEV framework with the Mellin transform gives rise to a better analytical result for 


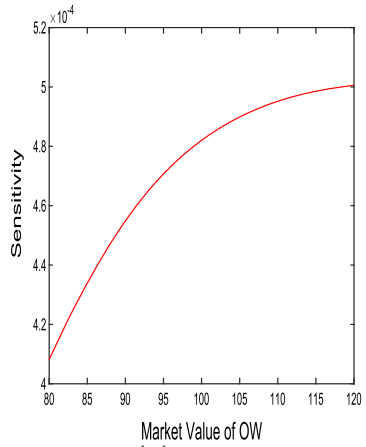

(a) $T=1$

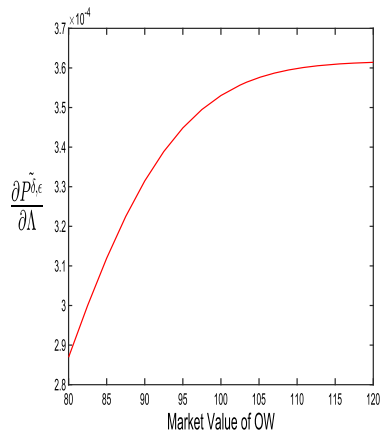

(b) $T=0.5$

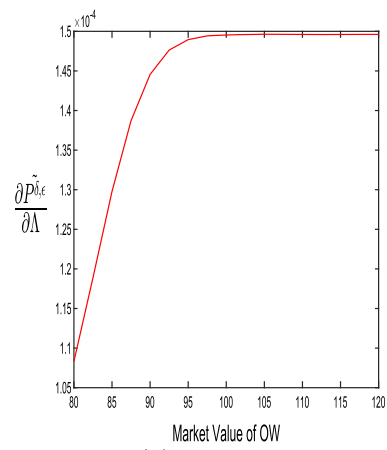

(c) $T=1 / 12$

Fig. 3 The Greek $\frac{\partial \tilde{P}^{\tilde{\partial}, \epsilon}}{\partial \Lambda}$ with $D^{*}=85$ for three time-to-maturities

option pricing with respect to the stability (or the accuracy) and the computing time than the Heston framework with the Fourier transform. This is, of course, important to practitioners for speedy calculation of more complicated derivatives and effective designing trading strategies.

Acknowledgements The work of S.-Y. Choi was supported by the National Research Foundation (NRF) of Korea (NRF-2019R1G1A1010278), The research of J.-H. Kim was supported by the NRF of Korea (NRF-2021R1A2C1004080), the research of J.-H. Yoon was supported by the NRF of Korea (NRF2017R1A5A1015722) and (NRF-2019R1A2C108931012) and the research of S. Veng was supported by a Higher Education Improvement Project grant funded by the Cambodian Government (IDA Credit No. 6221-KH).

\section{References}

Brychkov, Y. A. (1992). Multidimensional integral transformations. Boca Raton: CRC Press.

Choi, S.-Y., Kim, J.-H., \& Yoon, J.-H. (2016). The Heston model with stochastic elasticity of variance. Applied Stochastic Models in Business and Industry, 32(6), 804-824.

Delbaen, F., \& Shirakawa, H. (2002). A note on option pricing for the constant elasticity of variance model. Asia-Pacific Financial Markets, 9(2), 85-99.

Fouque, J.-P., Lorig, M., \& Sircar, R. (2016). Second order multiscale stochastic volatility asymptotics: Stochastic terminal layer analysis and calibration. Finance and Stochastics, 20(3), 543-588.

Fouque, J.-P., Papanicolaou, G., Sircar, R., \& Sølna, K. (2011). Multiscale stochastic volatility for equity, interest rate, and credit derivatives. Cambridge: Cambridge University Press.

Frontczak, R. (2013). Pricing options in jump diffusion models using Mellin transforms. Journal of Mathematical Finance, 3(03), 366.

Frontczak, R., \& Schöbel, R. (2010). On modified Mellin transforms, Gauss-Laguerre quadrature, and the valuation of American call options. Journal of Computational and Applied Mathematics, 234(5), 1559-1571.

Guardasoni, C., Rodrigo, M. R., \& Sanfelici, S. (2020). A Mellin transform approach to barrier option pricing. IMA Journal of Management Mathematics, 31(1), 49-67.

Heston, S. L. (1993). A closed-form solution for options with stochastic volatility with applications to bond and currency options. The Review of Financial Studies, 6(2), 327-343.

Hoffmann, P. S. (2018). Firm Value: Theory and Empirical Evidence. Rijeka: IntechOpen.

Hung, M. W., \& Liu, Y. H. (2005). Pricing vulnerable options in incomplete markets. Journal of Futures Markets: Futures, Options, and Other Derivative Products, 25(2), 135-170.

Jeon, J., Yoon, J. H., \& Kang, M. (2016). Valuing vulnerable geometric Asian options. Computers \& Mathematics with Applications, 71(2), 676-691. 
Jeon, J., Yoon, J. H., \& Kang, M. (2017). Pricing vulnerable path-dependent options using integral transforms. Journal of Computational and Applied Mathematics, 313, 259-272.

Johnson, H., \& Stulz, R. (1987). The pricing of options with default risk. The Journal of Finance, 42(2), 267-280.

Kim, J.-H., Lee, J., Zhu, S.-P., \& Yu, S.-H. (2014). A multiscale correction to the Black-Scholes formula. Applied Stochastic Models in Business and Industry, 30(6), 753-765.

Kim, J.-H., Yoon, J.-H., Lee, J., \& Choi, S.-Y. (2015). On the stochastic elasticity of variance diffusions. Economic Modelling, 51, 263-268.

Klein, P. (1996). Pricing Black-Scholes options with correlated credit risk. Journal of Banking \& Finance, 20(7), 1211-1229.

Krapivsky, P. L., \& Ben-Naim, E. (1994). Scaling and multiscaling in models of fragmentation. Physical Review E, 50(5), 3502.

Oksendal, B. (2013). Stochastic differential equations: An introduction with applications. Berlin: Springer.

Panini, R., \& Srivastav, R. P. (2004). Option pricing with Mellin transnforms. Mathematical and Computer Modelling, 40(1-2), 43-56.

Panini, R., \& Srivastav, R. P. (2005). Pricing perpetual options using Mellin transforms. Applied Mathematics Letters, 18(4), 471-474.

Yang, S.-J., Lee, M.-K., \& Kim, J.-H. (2014). Pricing vulnerable options under a stochastic volatility model. Applied Mathematics Letters, 34, 7-12.

Yoon, J.-H., \& Kim, J.-H. (2015). The pricing of vulnerable options with double Mellin transforms. Journal of Mathematical Analysis and Applications, 422(2), 838-857.

Yoon, J.-H., Kim, J.-H., \& Choi, S.-Y. (2013). Multiscale analysis of a perpetual American option with the stochastic elasticity of variance. Applied Mathematics Letters, 26(7), 670-675.

Yoon, J.-H., \& Park, C.-R. (2016). Pricing turbo warrants under stochastic elasticity of variance. Chaos, Solitons \& Fractals, 88, 107-118.

Publisher's Note Springer Nature remains neutral with regard to jurisdictional claims in published maps and institutional affiliations. 